\title{
Neuropathological features of Alzheimer's disease in non-demented parkinsonian patients
}

Parkinson's Disease Society Brain Bank, Institute of Neurology, London, UK $S$ E Daniel A J Lees

Correspondence to: Dr Daniel, Parkinson's Disease Society Brain Bank, Institute of Neurology, 1 Wakefield Street, London WC1N 1JP, UK

Received 7 January 1991 and in revised form 11 March

Accepted 26 March 1991.

\author{
S E Daniel, A J Lees
}

\begin{abstract}
Two patients with levodopa-responsive Parkinson's syndrome had numerous cortical and striatal senile plaques and some neurofibrillary tangles at necropsy. In addition neurons in the pars compacta of the substantia nigra were severely depleted but there were no Lewy bodies or other neuropathological changes to account for parkinsonism. Neither patient was demented. These pathological findings have not previously been described as a cause of Parkinson's syndrome without associated dementia of Alzheimer's disease type.
\end{abstract}

Most patients with a Parkinson's syndrome will be accurately diagnosed during life as having idiopathic Parkinson's disease. The pathological findings are characteristic and include degeneration of brain stem pigmented neurons with some of the remaining nerve cells containing Lewy bodies. ${ }^{1}$ However, even when strict clinical diagnostic criteria are applied ${ }^{2}$ misdiagnoses do occur and some patients thought clinically to have Parkinson's disease are found to have alternative pathologies at necropsy. Conversely, there are those patients with atypical clinical histories for whom the diagnosis of idiopathic Parkinson's disease is only established after death. ${ }^{3}$

The United Kingdom Parkinson's Disease Society Brain Bank receives donor tissue from parkinsonian patients who have been annually assessed by neurologists. From this material, $24 \%$ of patients with a clinical diagnosis of Parkinson's disease show different pathologies. ${ }^{4}$ Most frequently encountered are those conditions known to produce Parkinson's syndrome, for example, multiple system atrophy, progressive supranuclear palsy and postencephalitic Parkinson's disease. Less often, the underlying disease is unassociated with parkinsonism and may prove difficult to classify according to neuropathological criteria. We report two patients considered during life to have idiopathic Parkinson's disease in whom subsequent neuropathological examination revealed widespread cortical Alzheimer's disease change with numerous senile plaques and fewer neurofibrillary tangles although neither patient had been demented. There was a large number of plaques in the striatum, thalamus and hypothalamus. In the substantia nigra, pigmented nerve cells were severely depleted but there was no evidence of Lewy body disease. We suggest that involvement of the striatum and substantia nigra by Alzheimer's disease pathology was severe enough to cause clinical symptomatology indistinguishable from idiopathic Parkinson's disease.

\section{Case reports}

Case 1

A retired male supervisor presented at the age of 76 with a six month history of slowness, tremor, difficulty in walking, problems shaving and a stiff jaw. He also noticed difficulties in looking to one side when driving and on turning in bed. He complained of poor concentration and stated that people described him as having a "grim appearance". On examination he had facial hypomimia, a reduced steppage gait and bilateral cogwheel rigidity. There was no rest tremor. He was diagnosed as having idiopathic Parkinson's disease and started on Madopar $125 \mathrm{mg}$ four times daily, with definite improvement.

However, within six months of starting treatment he developed some involuntary facial movements and became increasingly depressed. Between 1980-83 he remained anxious and depressed and was treated with imipramine and selegiline. He would get up hourly at night to pass urine and was drowsy in the daytime. He was slow and stiff and had a shuffling gait with frequent "freezing". By 1986 he was still able to dress, feed and bathe himself but had a severe lowering of speech and frequent falls. He continued on Madopar. One year later he became incontinent of urine and was rather slow but mentally able. The patient died of bronchopneumonia in 1988 after a nine year illness. Necropsy examination was performed twelve hours after death and limited to the brain only.

\section{Neuropathological findings}

The whole brain weighed $1370 \mathrm{~g}$ unfixed and showed thickening of the leptomeninges over the vertex. The brain was cut mid-sagittally; the right half was frozen for chemical analysis while the left half was fixed for six weeks in $10 \%$ neutral formalin and available for neuropathological examination. Coronal slices of the cerebral hemisphere showed a slightly dilated lateral ventricle. Pigmentation was reduced in the substantia nigra and locus ceruleus. Tissue blocks were taken from frontal, temporal, insular, parietal and occipital cortices, hippocampus, striatum, thalamus, hypothalamus, amygdala, substantia innominata, cerebellar vermis and hemisphere, midbrain, pons and medulla. Sections were stained with haematoxylin-eosin ( $\mathrm{HE}$ ), luxol fast bluecresyl violet, modified Bielschowsky silver 
impregnation and Congo red. Plaque counts in several microscopic fields encompassing $1 \mathrm{~mm}^{2}$ were made on $7 \mu$ Bielschowsky stained sections of neocortex (frontal, temporal, parietal, occipital) and hippocampus. In selected regions the following antigens were looked for immunocytochemically using a biotin-streptavidin system: Tau-1 (Sigma, monoclonal; 1:1000), amyloid A4 protein (Dr BH Anderton, polyclonal 12-28 residues), ubiquitin (Dako, polyclonal 1:400), glial fibrillary acidic protein (Dako, polyclonal 1:1000).

In the cerebral cortex there was a large number of neurofibrillary tangles and senile plaques. Tangles were argyrophilic and immunostained with antisera to Tau-1 and ubiquitin. They were of typical appearance with a triangular or looped arrangement of thickened neurofibrils occupying the neuronal cytoplasm. Plaques of different morphology were identified: 1) Belonged to the classic variety ${ }^{5}$ with primitive, mature and burnt-out types. The neuritic processes were argyrophilic and immunoreactive with Tau-1, ubiquitin and A4 protein. The amyloid core was congophilic and A4 reactive. 2) Diffuse plaques ${ }^{6}$ lacked a central amyloid core and were composed of granular argyrophilic areas which were immunostained with A4 but not by Tau-1 or ubiquitin antibody. The number of neocortical senile plaques per $\mathrm{mm}^{2}$ exceeded that required for a histological diagnosis of Alzheimer's disease. ${ }^{7}$ In the hippocampus many neurons showed granulovacuolar degeneration and there were Hirano bodies present in addition to plaques and tangles.

Senile plaques were numerous in the caudate (fig 1), putamen, thalamus, hypothalamus, substantia innominata and amygdaloid nuclear type plaques were approximately equally represented. Large numbers of globose neurofibrillary tangles were identified in neurons of the thalamus, hypothalamus, substantia innominata and amygdala; occasional large neurons of the caudate and putamen also contained tangles. The globus pallidus contained neither plaques nor tangles.

In the brain stem there were a few plaques in the periaqueductal region and pontine base. Neurofibrillary tangles were quite frequent and found at the following sites: ventral tegmental

Figure 1 Caudate nucleus at the level of nucleus accumbens. In this field there are numerous plaques (some arrowed), varying considerably in size and mainly of the diffuse variety. Modified diffuse variety. Modified
Bielschowsky stain, $\times 12$. (case 1) complex. Diffuse (fig 2a) and classical (fig 2b)

area, substantia nigra, oculomotor nucleus, cuneiform nucleus, locus ceruleus, superior central nucleus and median raphe.

The substantia nigra showed severe depletion of pigmented neurons (fig 3 ) with some extracellular pigment and an increase of microglial cells and astrocytes. In the locus ceruleus there was also a loss of pigmented nerve cells, and free-lying pigment. Lewy bodies were looked for using $\mathrm{HE}$ and ubiquitin staining but none were identified at any site.

Amyloid angiopathy involved a large proportion of leptomeningeal and cortical vessels in the cerebrum and cerebellum and also occasional arterioles in the striatum. In some instances the vascular amyloid had provoked secondary hyalinisation of the vessel wall and reactive astrocytosis in the surrounding neuropil.

\section{Case 2}

A 66 year old woman presented with tremor in the leg and subsequently developed severe slowing of all voluntary movements, with great difficulty in rising from a chair or turning
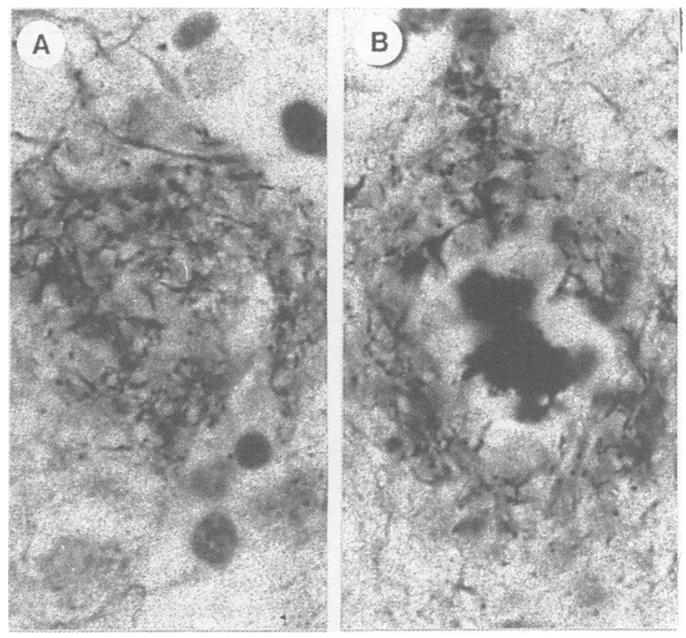

Figure 2 Striatal senile plaques demonstrated with the modified Bielschowsky stain: a) plaque of diffuse variety in the caudate nucleus; $b$ ) classical neuritic type plaque with a mature appearance in the putamen, $\times 1500$. (case 1)

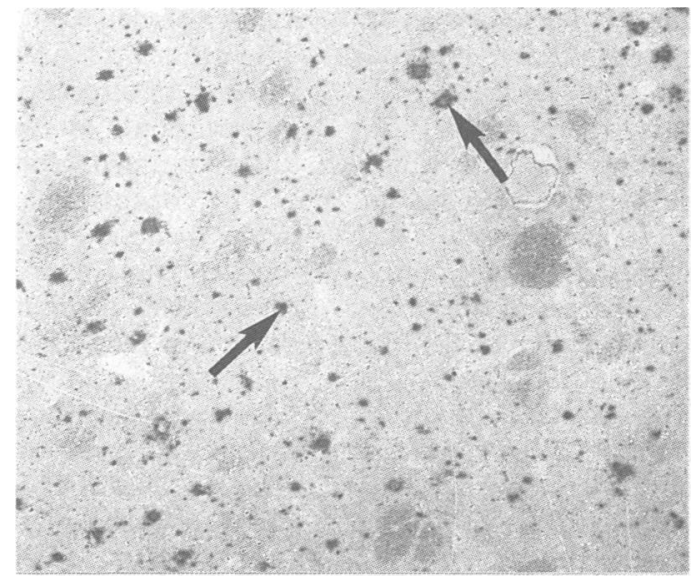

Figure 3 Lateral substantia nigra showing only one remaining nerve cell in this field (arrowhead) and remaining nerve cell in this field (arrowhead) and
reactive astrocytes (some arrowed). $H E, \times 100$. (case 1) 
Figure 4 Insular cortex showing senile plaques extending from the subpial surface (top) to the subcortical white matter. Modified Bielschowsky stain, $\times 12.5$. (case 2)

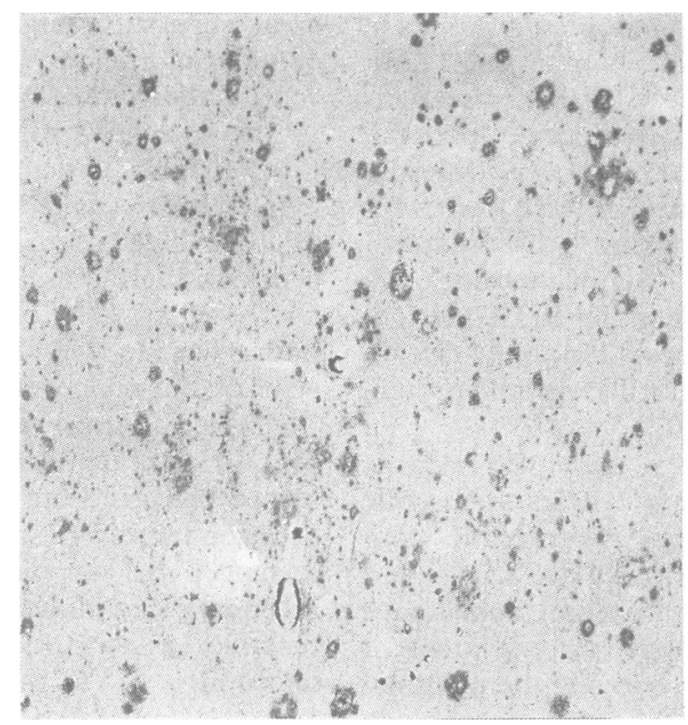

rapidly when walking. A diagnosis of idiopathic Parkinson's disease was made and levodopa was started two years after her initial presentation, with a good response. Within a year of starting treatment involuntary movements of the face were noted. Six years after the onset of her disease she was found to have mild rigidity, bilateral bradykinesia with extremely poor balance and marked slowness of dressing; there was no tremor. Walking was limited to about 100 yards and was without arm-swing. Both plantar responses were reported as being extensor. A Beck Depression score of 22 was recorded and a Folstein Mini-Mental score of 26. Levodopa-induced chorea continued and nine years after the onset of her illness the patient became severely immobile with urinary incontinence and slurred speech. There were abnormalities of her respiratory rate with periods of apnoea and shallow, rapid respirations. She died in 1989 , ten years after the onset of her disease. General necropsy found bronchopneumonia.

\section{Neuropathological findings}

The brain weighed $1115 \mathrm{~g}$ before fixation. Externally the leptomeninges were thickened and there was fronto-parietal atrophy. The left hemibrain was formalin-fixed for morphological examination. Coronal slices of the cerebrum showed moderate dilatation of the ventricular system. Pigment was scant in the substantia nigra and locus ceruleus. Tissue was prepared for microscopy using the methods described for case 1 .

Sections of the cerebral cortex (fig 4), hippocampal formation, substantia innominata and amygdala showed numerous senile plaques and fewer neurofibrillary tangles; there was granulovacuolar degeneration of pyramidal hippocampal neurons. In all areas of the cortex the number of senile plaques per $\mathrm{mm}^{2}$ fulfilled the histological criteria for the diagnosis of Alzheimer's disease. ${ }^{7}$ The caudate, putamen, thalamus and hypothalamus also contained senile plaques and very few neurofibrillary tangles. These nuclei appeared slightly atrophic with an increase of astrocytes.
In the brain stem there were several neuritic plaques in the periaqueductal region. At the following sites a few nerve cells contained neurofibrillary tangles: superior colliculus, griseum centrale mesencephale, oculomotor nucleus, ventral tegmental area and locus ceruleus. In the substantia nigra pigmented neurons were severely depleted; free pigment granules and occasional macrophages were present. There was extracellular pigment and a reduction of cell number in the locus ceruleus. Lewy bodies were not found in either the pigmented brain stem nuclei or sites elsewhere.

Additional pathology was confined to the vasculature where there was mild congophilic angiopathy of a few small cortical vessels and arteriolosclerosis in the deep grey nuclei.

\section{Discussion}

In both cases the clinical diagnosis of idiopathic Parkinson's disease was not supported by the neuropathological examination. We attribute the parkinsonian syndrome to severe nigral nerve cell loss and the large number of striatal plaques. Although there was no dementia the microscopic appearances of the hippocampus were characteristic of Alzheimer's disease and furthermore, the number of neocortical plaques fulfilled the currently accepted morphological criteria for this diagnosis. ${ }^{7} \mathrm{We}$ use the term "Alzheimer's disease-type change" to emphasise that mentation in our patients was well-preserved and thus distinguish them from those individuals with a parkinsonian syndrome plus dementia in whom the sole pathology is that of Alzheimer's disease (personal observations, ${ }^{8}{ }^{9}$ ). The occurrence of large numbers of plaques and tangles in the brains of non-demented elderly people is increasingly recognised ${ }^{1011}$ with the use of special silver techniques and immunostaining for A4 protein. It remains uncertain, however, whether such individuals represent cases of preclinical Alzheimer's disease or if they would never have developed cognitive impairment. Compensatory mechanisms might occur and an individual may not show signs of dementia if they have a large brain ${ }^{12}$ or if the "amyloid load" is easily accommodated. ${ }^{13}$ Alternatively, it appears that while cortical neurofibrillary tangles are closely correlated with dementia ${ }^{1415}$ the senile plaques, in particular the diffuse type, may correlate with memory recall ${ }^{16}$ but not necessarily dementia. ${ }^{15}$ The diffuse plaque may evolve to the classical variety ${ }^{11}$ or alternatively plaque types might be independent of each other and represent different morphological substrates of disease.

The occurrence of senile plaques and neurofibrillary tangles in the striatum and diencephalon of patients with Alzheimer's disease is well-described ${ }^{17-19}$ but plaques, and in particular tangles, are exceptionally rare as an incidental finding in these nuclei. ${ }^{17}{ }^{19}$ Nigral neuronal depletion also occurs in Alzheimer's disease but is not usually severe, ${ }^{2021}$ and unlike cell loss in the locus ceruleus ${ }^{22}$ does not correlate with severity of dementia.

In our patients the nigral nerve cell loss was 
comparable to that occurring in idiopathic Parkinson's disease but there was no evidence of Lewy bodies; alternative diagnoses such as postencephalitic parkinsonism or progressive supranuclear palsy are not supported by the histological findings. While there were brain stem tangles, several sites of predilection in progressive supranuclear palsy were spared and there was no involvement of the following nuclei: globus pallidus, corpus Luysii, basis pons, inferior olive and dentate. We believe that the depletion of pigmented nigral neurons is related to the cortical pathology and may have been exacerbated by the striatal plaques. Both patients improved with levodopa and such an observation has also been made for the rigidity in Alzheimer's dementia. ${ }^{23}$ Whether the condition we report represents an extrapyramidal syndrome in preclinical Alzheimer's disease or a hitherto unrecognised disease entity remains uncertain. Similar cases have not been reported in the literature and further studies are required to clarify the characteristics of this clinicopathological entity.

We thank Miss Siobhan Blankson and Miss Linda Kilford for the histological preparations and Miss Rita Nani for her secretarial assistance. We are grateful to the MRC Alzheimer's Disease Brain Bank for performing immunostaining for A4 protein. Dr SE Daniel is funded by the Parkinson's Disease Society, UK.

1 Oppenheimer DR. Diseases of the basal ganglia, cerebellum and motor neurons. In: Adams $\mathrm{JH}$, Corsellis JAN, Duchen LW, eds. Greenfield's Neuropathology, 4th ed. New York: Wiley, 1984:702.

2 Gibb WRG, Lees AJ. The significance of the Lewy body in the diagnosis of idiopathic Parkinson's disease. Neuropathology and Applied Neurobiology. 1989;15:27-44.

3 Sage JI, Miller DC, Golbe LI, Walters A, Duvoisin RC. Clinically atypical expression of pathologically typical Lewy body parkinsonism. Clinical Neuropatholgy. Lewy body
$1990 ; 13: 36-47$.

4 Hughes AJ, Daniel SE, Kilford L, Lees AJ. The accuracy of clinical diagnosis of idiopathic Parkinson's disease: a clinico-pathological study of 100 cases. J Neurol Neurosurg Psychiatry (in press)

5 Wisniewski HM, Terry RD. Re-examination of the pathogenesis of the senile plaque. In: Zimmerman HM, ed Progress in Neurology. New York: Grune and Stratton, 1973:2-26
6 Yamaguchi H, Hirai S, Marimatsu M, Shoji M, Ihara Y. A variety of cerebral amyloid deposits in the brains of the Alzheimer-type dementia demonstrated by $\beta$ protein immunostaining. Acta Neuropathol. 1988;76:541-9.

7 Khachaturian ZS. Diagnosis of Alzheimer's disease. Arch Neurol 1985;42:1097-105.

8 Joachim CL, Morris JH, Selkoe DJ. Clinically diagnosed Alzheimer's disease: autopsy results in 150 cases. Ann Neurol 1988;24:50-6

9 Csanda E, Papp MI, Tarczy M, Takats A. Some possible histopathological correlates of dementia in parkinsonian patients. In: Batistin L, Gerstenbrand F, eds. Aging brain and dementia: new trends in diagnosis and therapy. New York: Wiley-Liss, 1990:119-32.

10 Wisniewski HM, Rabe A, Zigman W, Silverman W. Neuropathological diagnosis of Alzheimer's disease. $j$ Neuropathol Exp Neurol 1989;48:606-9.

11 Mann DMA, Brown AMT, Prinja D, Jones D, Davies CA. A morphological analysis of senile plaques in the brains of non-demented persons of different ages using silver, immunter techniques. Neuropath Appl Neurobiol 1990;16:17-25.

12 Katzman R, Terry R, DeTeresa R, Brown T, Davies P, Fuld P, Renbing X, Peck A. Clinical, pathological and neurochemical changes in dementia: a subgroup with preserved chemical changes in dementia: a subgroup with preserved mental status and numerol

13 Tomlinson BE. Dementia and the deposition of amyloid in the brain. Neuropathology (Suppl). 1986;3:1-12.

14 Wilcock GK, Esiri MM. Plaques, tangles and dementia. $J$ Neurological Sciences 1982;56:343-56.

15 Crystal H, Dickson D, Fuld P et al. Clinico-pathologic studies in dementia: non-demented subjects with pathologically confirmed Alzheimer's disease. Neurology. 1988;38:1682-7.

16 Fuld PA, Dickson D, Crystal H, Aronson MK. Primitive plaques and memory dysfunction in normal and impaired plaques and memory dysfunction in normal and im

17 Braak H, Braak E. Alzheimer's disease: striatal amyloid deposits and neurofibrillary changes. $J$ Neuropath Exper Neurol 1990;49:215-24.

18 Rudelli RD, Ambler MW, Wisniewski HM. Morphology and distribution of Alzheimer neuritic (senile) and amyloid plaques in striatum and diencephalon. Acto Neuropathol 1984;64:273-81

19 Suenaga T, Hirano A, Llena JF, Yen S-H, Dickson DN Modified Bielschowsky stain and immunohistochemica studies on striatal plaques in Alzheimer's disease. Acta Neuropathol 1990;80:280-6.

20 Gibb WRG, Mountioy CQ, Mann DMA, Lees AJ. A pathological study of the association between Lewy body disease and Alzheimer's disease. J Neurol Neurosurg Psychiatry 1989;52:701-8.

21 Rinne JO, Rummukainen J, Paljarvi L, Sako E, Molsa P Rinne UK. Neuronal loss in the substantia nigra in patients with Alzheimer's disease and Parkinson's disease patients with Alzheimer's disease and Parkinson's disease in relation to extrapyramidal symptoms and dementia. In: disease and related disorders. New York: Alan R Liss, 1989:325-52.

22 Tomlinson BE, Irving D, Blessed G. Cell loss in the locus coeruleus in senile dementia of Alzheimer's type. J Neurol Sci 1981;49:419-28.

23 Bennett RG, Greenbough, III WB, Gloth, III FM, Lindsay JM, Zelesnick L. Extrapyramidal signs in dementia of Alzheimer type. Lancet 1989;ii:1392. 